German Business Panel

\title{
Wie Unternehmen des Baugewerbes die Corona-Krise bewerten
}


Die Ergebnisse in diesem Dokument basieren auf den Auswertungen der Corona-Befragung des German Business Panel. Sie werden fortlaufend erweitert und aktualisiert. Weitere Informationen: www.gbpanel.org

Die methodischen und inhaltlichen Grundlagen der Studie finden Sie in unserem Working Paper: The German Business Panel: Insights on corporate taxation and accounting during the COVID-19 pandemic

\section{Autoren}

Prof. Dr. Jannis Bischof, Prof. Dr. Philipp Dörrenberg, Johannes Gaul, Clemens Lauer, Dr. Davud Rostam-Afschar, Prof. Dr. Dirk Simons, Prof. Dr. Johannes Voget

Universität Mannheim

\section{German Business Panel}

\section{Das German Business Panel ist ein langfristiges Befragungspanel des DFG-geförderten überregionalen Projektes „Accounting for Transparency".}

Wir erheben systematisch und repräsentativ, wie Unternehmen unterschiedlicher Größenordnung Fragen der Unternehmenstransparenz einschätzen. Erhoben wird auch, wie sich die zunehmende Bereitstellung von Informationen sowie die zunehmende Regulierung in den Bereichen Rechnungswesen und Besteuerung auf Entscheidungen in Unternehmen und die Öffentlichkeit auswirken. Als umfassendes, langfristig angelegtes Befragungspanel liefern wir Daten in einer bisher nicht dagewesenen Qualität für die Forschung zu Unternehmenstransparenz und tragen dazu bei, Regulierung und Wirtschaft zu verbessern. Das German Business Panel ist Teil des Sonderforschungsbereichs „TRR 266 Accounting for Transparency".

Leitung des German Business Panel:

Principal Investigators

(wissenschaftliche Projektleitung):

Prof. Dr. Jannis Bischof

Universität Mannheim

jbischof@uni-mannheim.de

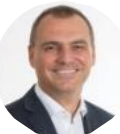

Prof. Dr. Dirk Simons

Universität Mannheim

simons@uni-mannheim.de

Prof. Dr. Johannes Voget

Universität Mannheim

voget@uni-mannheim.de
Research Fellow

(wissenschaftliche Begleitung):

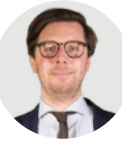

Prof. Dr. Philipp Dörrenberg

Universität Mannheim

doerrenberg@uni-mannheim.de

Projektmanager:

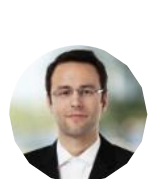

Dr. Davud Rostam-Afschar

Universität Mannheim

rostam-afschar@uni-mannheim.de

www.gbpanel.org | gbpinfo@mail.uni-mannheim.de

\section{TRR 266 Accounting for Transparency}

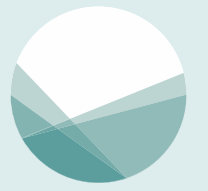

\section{Der TRR 266 Accounting for Transparency ist ein von der Deutschen Forschungsgemeinschaft (DFG) geförderter überregionaler Sonderforschungsbereich.}

Unser Team von engagierten Forschenden untersucht, wie Rechnungswesen und Besteuerung die Transparenz von Unternehmen beeinflussen und wie sich Regulierungen und Unternehmenstransparenz auf Wirtschaft und Gesellschaft auswirken. Ziel ist es, eine wirksame Regulierung für Unternehmenstransparenz und ein transparentes Steuersystem zu entwickeln. In unserer eigenen Forschung gewährleisten wir die Transparenz ebenfalls.

Am TRR 266 sind mehr als 80 Wissenschaftlerinnen und Wissenschaftler beteiligt von den Hauptstandorten: Universität Paderborn (Sprecherhochschule), Humboldt-Universität zu Berlin und Universität Mannheim, zudem Forscherinnen und Forscher von der Ludwig-Maximilians-Universität München sowie der ESMT Berlin, der Frankfurt School of Finance \& Management, der Goethe-Universität Frankfurt am Main, und der WHU - Otto Beisheim School of Management. 


\section{Die Corona-Umfrage des German Business Panel}

Die Corona-Krise stellt Unternehmen in Deutschland vor beispiellose Herausforderungen. Das German Business Panel (GBP) der Universität Mannheim untersucht insbesondere folgende Fragen:

1. Wie entwickeln sich Umsatz und Gewinn deutscher Unternehmen während der Krise?

2. Haben staatliche Hilfsleistungen Unternehmen in der Krise erreicht?

3. Welche staatlichen Leistungen haben Unternehmen in welchem Umfang in Anspruch genommen?

4. Hätten Unternehmen auch ohne staatliche Hilfe die Krise überstanden?

5. Welche strategischen Entscheidungen haben Unternehmen als Reaktion auf die Krise getroffen?

6. Welche Erwartungen haben Unternehmen in Bezug auf die Überlebenswahrscheinlichkeit von Unternehmen in ihrer Branche?

7. Für welchen Zeithorizont planen Unternehmen mit Einschränkungen aufgrund der Corona-Krise?

Die Ergebnisse der GBP-Corona-Befragung geben Aufschluss darüber, wie zielgerichtet und wie wirksam die staatlichen Hilfsmaßnahmen für Unternehmen waren.

\section{Baugewerbe kommt besser durch die Corona-Krise}

- Im Vergleich zu anderen Industrien schneiden Unternehmen des Baugewerbes in wichtigen finanziellen Kennzahlen besser ab. Die wirtschaftlichen Einbußen bei Gewinn und Umsatz fallen bei diesen Unternehmen deutlich geringer aus als bei Unternehmen der anderen Industrien. Unternehmen des Baugewerbes erwarten dennoch ein geringeres BIP-Wachstum in 2021.

- Im Baugewerbe schneiden gerade mittelgroße Unternehmen gemessen an der Umsatzveränderung insgesamt besser ab. Jedoch ist im Baugewerbe der Unterschied zwischen den Größenklassen insgesamt geringer als innerhalb der anderen Industrien.

- Bei der Inanspruchnahme von staatlichen Maßnahmen gibt es erhebliche Unterschiede zwischen Unternehmen des Baugewerbes und Unternehmen der anderen Industrien. Sowohl bei den nicht-steuerlichen als auch bei den steuerlichen Maßnahmen haben durchschnittlich deutlich weniger Unternehmen des Baugewerbes Hilfen beantragt oder erhalten. Auch berichten weniger Unternehmen des Baugewerbes von konkreten Hürden bei der Beantragung.

- Trotzdem geht im Baugewerbe ein höherer Anteil der Unternehmen davon aus, dass die staatlichen Maßnahmen nicht ausreichen, um die Corona-Krise zu überleben. 
- Auch bei weiteren internen Maßnahmen zur Deckung der Mehrbelastung durch die Corona-Krise berichten durchschnittlich weniger Unternehmen des Baugewerbes, solche Maßnahmen ergriffen zu haben. Die Reduktion von geplanten Löhnen und Boni ist für Unternehmen des Baugewerbes die wichtigste Maßnahme zur Kompensation der Mehrbelastung.

- Eine detailliertere Unterscheidung zwischen Ausbaugewerbe und Bauhauptgewerbe legt Unterschiede in der pandemiebedingten Belastung zwischen den jeweiligen Branchen offen.

- Betrachtet nach zusammengefassten Wirtschaftsgruppen dreht sich der insgesamt beobachtbare Zusammenhang zwischen Unternehmensgröße und erwarteter Umsatzveränderung für das Bauhauptgewerbe um. Insbesondere kleine Unternehmen rechnen mit Umsatzzuwächsen, große Unternehmen immerhin mit einem leichten Umsatzwachstum.

- Demgegenüber erwarten kleine Unternehmen des Ausbaugewerbes im Besonderen und Unternehmen der Ausbaubranche insgesamt stärkere Umsatzeinbrüche.

- Die unterschiedliche Belastung spiegelt sich ebenso in der Inanspruchnahme staatlicher Maßnahmen wider. Nicht-steuerliche Maßnahmen wurden von Betrieben des Ausbaugewerbes stärker in Anspruch genommen als von denen des Bauhauptgewerbes. Das betrifft insbesondere die Corona-Soforthilfe sowie das Kurzarbeitergeld. In der Inanspruchnahme steuerlicher Maßnahmen wiederum sind nur sehr geringe Unterschiede innerhalb der Baubranche sichtbar.

- Etwa zwei Drittel der Betriebe in Ausbau- und Hauptbaugewerbe bezeichnen die bisherigen Maßnahmen als unzureichend.

- Beinahe jedes fünfte Unternehmen des Ausbaugewerbes gibt an, Mitarbeiter als Mittel zur Krisenbewältigung entlassen zu haben. Innerhalb des Bauhauptgewerbes trifft dies auf weniger als $10 \%$ der Betriebe zu.

\section{Fazit}

Die wirtschaftliche Situation der Unternehmen stellt sich im Baugewerbe besser dar als in den anderen Industrien. Gleichzeitig haben die Unternehmen des Baugewerbes weniger staatliche Maßnahmen in Anspruch genommen. Zur Kompensation der Mehrbelastung greifen die meisten von ihnen auf eine Reduktion von Löhnen und Boni zurück. Innerhalb des Baugewerbes werden zwischen Ausbau- und Bauhauptgewerbe starke Unterschiede in der krisenbedingten Belastung deutlich. Dies betrifft die Erwartungen zur Umsatzentwicklung sowie die Inanspruchnahme staatlicher Maßnahmen. 


\section{I -Baugewerbe im Vergleich zu anderen Industrien}

Die Ergebnisse in allen Abschnitten des Berichts basieren auf zwei Befragungen aus den Zeiträumen von Juli bis September 2020 sowie von November 2020 bis März 2021. Die erste Befragung umfasst mehr als 10.000 abgeschlossene Interviews, davon 748 aus dem Baugewerbe. Die zweite Befragung bietet 9.093 abgeschlossene Interviews, davon 462 aus dem Baugewerbe (letzter Stand: 30.03.2021). Die Befragungsergebnisse des Baugewerbes sind mit den Daten des Unternehmensregisters des Statistischen Bundesamts (2019) zur Anzahl der Unternehmen in Abhängigkeit von Mitarbeitergrößenklassen gewichtet.

\section{Kennzahlen: Baugewerbe vs. andere Industrien}

Die folgende Tabelle zeigt, wie sich wesentliche Kennzahlen innerhalb des Baugewerbes im Vergleich zu anderen Industrien verändert haben - basierend auf den Berichten der teilnehmenden Unternehmen. Die Veränderungen ergeben sich aus einem Vergleich der Kennzahlen zum Jahresbeginn 2020. Die jeweiligen Werte in Klammern beziehen sich hier und im Folgenden auf die Antworten der Unternehmen in der ersten Befragungswelle im Sommer 2020. Es zeigt sich, dass die Unternehmen des Baugewerbes über alle Kennzahlen hinweg deutlich besser durch die Krise gekommen sind als die Unternehmen der anderen Industrien. Im Gegensatz zu den Unternehmen der anderen Industrien gaben diese beim Umsatz nur leicht negative Veränderungen an. Die Anzahl der Mitarbeiter konnte das Baugewerbe gegenüber dem Vorjahr trotz Corona-Krise im Durchschnitt sogar erhöhen.

Tabelle 1: Unternehmen des Baugewerbes überstehen Corona-Krise besser

\begin{tabular}{|c|c|c|c|c|c|c|}
\hline & \multicolumn{3}{|c|}{ Baugewerbe } & \multicolumn{3}{|c|}{ Andere Industrien } \\
\hline & Durchschnitt & S.F. & N & Durchschnitt & S.F. & N \\
\hline $\begin{array}{l}\text { Umsatzveränderung ggü. } \\
\text { Vorjahr (\%) }\end{array}$ & $\begin{array}{l}-1,9 \\
(-1,7)\end{array}$ & $\begin{array}{c}2,58 \\
(2,96)\end{array}$ & $\begin{array}{c}434 \\
(653)\end{array}$ & $\begin{array}{l}-22,5 \\
(-22,2)\end{array}$ & $\begin{array}{c}1 \\
(1)\end{array}$ & $\begin{array}{c}8.632 \\
(10.990)\end{array}$ \\
\hline $\begin{array}{l}\text { Gewinnveränderung ggü. } \\
\text { Vorjahr (\%) }\end{array}$ & $\begin{array}{c}-1 \\
(-0,8)\end{array}$ & $\begin{array}{c}2,61 \\
(2,94)\end{array}$ & $\begin{array}{l}427 \\
(623)\end{array}$ & $\begin{array}{l}-22,9 \\
(-19,4)\end{array}$ & $\begin{array}{c}1,01 \\
(1,03)\end{array}$ & $\begin{array}{c}8.315 \\
(10.362)\end{array}$ \\
\hline $\begin{array}{l}\text { Liquiditätsveränderung ggü. } \\
\text { Vorjahr (\%) }\end{array}$ & $\begin{array}{l}-0,6 \\
(2)\end{array}$ & $\begin{array}{c}2,51 \\
(2,76)\end{array}$ & $\begin{array}{c}431 \\
(643)\end{array}$ & $\begin{array}{l}-15,1 \\
(-10,2)\end{array}$ & $\begin{array}{c}1,01 \\
(0,95)\end{array}$ & $\begin{array}{c}8.321 \\
(10.543)\end{array}$ \\
\hline $\begin{array}{l}\text { Veränderung der } \\
\text { Mitarbeiteranzahl ggü. } \\
\text { Vorjahr }\end{array}$ & $\begin{array}{c}2,1 \\
(6,6)\end{array}$ & $\begin{array}{c}2,04 \\
(2,57)\end{array}$ & $\begin{array}{c}436 \\
(647)\end{array}$ & $\begin{array}{l}-2,5 \\
(1,6)\end{array}$ & $\begin{array}{c}0,84 \\
(0,71)\end{array}$ & $\begin{array}{c}8.411 \\
(10.807)\end{array}$ \\
\hline
\end{tabular}

S.F. bezeichnet den Standardfehler des Mittelwerts, N die Zahl der Beobachtungen. Die Werte in Klammern beziehen sich auf die jeweiligen Ergebnisse der ersten Befragungswelle. 
Tabelle 2 zeigt, wie stark die teilnehmenden Unternehmen die allgemeine Unsicherheit, mit der sie konfrontiert sind, bewerten. Sie stellt außerdem die erwartete Überlebensrate in der Branche und die erwartete Veränderung des Bruttoinlandsprodukts (BIP) in 2021 dar. Außerdem zeigt sie, wie zufrieden die befragten Unternehmen mit den staatlichen Maßnahmen sind. Dabei fällt Folgendes auf: Trotz geringerer Unsicherheit, einer höheren erwarteten Überlebensrate im Baugewerbe und besserer finanzieller Kennzahlen (Tabelle 1), fällt das erwartete BIP-Wachstum in 2021 deutlich schlechter aus als in den anderen Industrien. Hier hat sich die Situation im Vergleich zur ersten Befragungswelle umgekehrt. Damals lag die Erwartung des Baugewerbes noch über der Erwartung der anderen Industrien.

Tabelle 2: Geringere Unsicherheit im Baugewerbe, jedoch schlechtere BIP-Erwartung

Baugewerbe

Durchschnitt
Andere Industrien

\begin{tabular}{lccc|ccc} 
& Durchschnitt & S.F. & $N$ & Durchschnitt & S.F. & $N$ \\
\hline Allgemeine Unsicherheit & 3,6 & 0,07 & 434 & 3,9 & 0,03 & 7.998 \\
(Skala: 1-5) & $(\mathrm{n} / \mathrm{a})$ & $(\mathrm{n} / \mathrm{a})$ & $(\mathrm{n} / \mathrm{a})$ & $(\mathrm{n} / \mathrm{a})$ & $(\mathrm{n} / \mathrm{a})$ & $(\mathrm{n} / \mathrm{a})$ \\
Erwartete Überlebensrate in & 80,6 & 1,36 & 459 & 71,5 & 0,52 & 8.470 \\
eigener Branche (\%) & $(84,4)$ & $(1,46)$ & $(637)$ & $(76,8)$ & $(0,46)$ & $(8.827)$ \\
Erwartete BIP-Veränderung in & $-8,9$ & 0,81 & 335 & $-7,3$ & 0,27 & 6.271 \\
2021 (\%) & $(-0,8)$ & $(0,38)$ & $(574)$ & $(-1,2)$ & $(0,12)$ & $(8.515)$ \\
Zufriedenheit mit staatl. & & & & & & \\
$\begin{array}{l}\text { Maßnahmen ggü. Vorjahr } \\
\text { (Skala: 0-4) }\end{array}$ & 1,6 & 0,1 & 198 & 1,6 & 0,03 & 5.031 \\
& $(\mathrm{n} / \mathrm{a})$ & $(\mathrm{n} / \mathrm{a})$ & $(\mathrm{n} / \mathrm{a})$ & $(\mathrm{n} / \mathrm{a})$ & $(\mathrm{n} / \mathrm{a})$ & $(\mathrm{n} / \mathrm{a})$ \\
\hline
\end{tabular}

S.F. bezeichnet den Standardfehler des Mittelwerts, N die Zahl der Beobachtungen. Die Werte in Klammern beziehen sich auf die jeweiligen Ergebnisse der ersten Befragungswelle.

Vergleicht man die Umsatzveränderung der Unternehmen nach Mitarbeiteranzahl im Baugewerbe mit den anderen Industrien, ergeben sich deutliche Unterschiede. Stehen bei den anderen Industrien die kleinen Unternehmen mit bis zu fünf Mitarbeitern deutlich am schlechtesten dar, gefolgt von den mittelgroßen Unternehmen, so ist die Lage bei den Unternehmen des Baugewerbes gemischt. Hier schneiden die mittelgroßen Unternehmen mit einem durchschnittlichen Umsatzwachstum von 3,3 \% am besten ab, wohingegen die kleinen und großen Unternehmen eine ähnlich negative Umsatzentwicklung verzeichnen. 
Tabelle 3: Kleine Unternehmen auch im Baugewerbe härter getroffen

Baugewerbe

Andere Industrien

\begin{tabular}{lccc|ccc}
$\begin{array}{l}\text { Umsatzveränderung ggü. } \\
\text { Vorjahr (\%) }\end{array}$ & Durchschnitt & S.F. & N & Durchschnitt & S.F. & N \\
\hline Null bis fünf Mitarbeiter & $-2,3$ & 2,91 & 246 & $-23,3$ & 1,1 & 6.105 \\
& $(-2,6)$ & $(3,34)$ & $(371)$ & $(-23,2)$ & $(1,18)$ & $(6.239)$ \\
Sechs bis 19 Mitarbeiter & 3,3 & 4,16 & 93 & $-15,4$ & 1,95 & 1.146 \\
& $(7,7)$ & $(3,76)$ & $(120)$ & $(-21)$ & $(2,27)$ & $(1.318)$ \\
Mehr als 20 Mitarbeiter & $-2,5$ & 3,55 & 95 & $-13,3$ & 1,76 & 1.639 \\
& $(2,3)$ & $(2,51)$ & $(161)$ & $(-15,1)$ & $(1,99)$ & $(1.880)$ \\
\hline
\end{tabular}

S.F. bezeichnet den Standardfehler des Mittelwerts, N die Zahl der Beobachtungen. Die Werte in Klammern beziehen sich auf die jeweiligen Ergebnisse der ersten Befragungswelle.

\section{Inanspruchnahme von staatlichen Maßnahmen}

Bei der Inanspruchnahme von staatlichen Maßnahmen unterscheiden sich die Unternehmen des Baugewerbes ebenfalls deutlich von den Unternehmen der anderen Industrien. Während die Mehrheit der Unternehmen (60\%) in den anderen Industrien nicht-steuerliche staatliche Maßnahmen beantragt oder erhalten hat, sind es im Baugewerbe nur knapp die Hälfte (44 \%) der Unternehmen.

Durchschnittlich weniger Unternehmen des Baugewerbes haben nicht-steuerliche staatliche Maßnahmen beantragt oder erhalten

Nicht-steuerliche Maßnahmen beantragt oder erhalten:

\section{$44 \% \quad 60 \%$}

des Baugewerbes

der anderen Industrien

Hinsichtlich der Relevanz der in Anspruch genommenen, nicht-steuerlichen Maßnahmen zeigt sich im Baugewerbe und in den anderen Industrien ein ähnliches Bild. Tabelle 4 zeigt die vier staatlichen Maßnahmen, die am häufigsten von Unternehmen genannt bzw. in Anspruch genommen wurden, sowie den jeweiligen Anteil der Unternehmen. Auch im Baugewerbe stellt die Soforthilfe Corona die wichtigste staatliche Maßnahme dar (29,5\%), gefolgt von der Kurzarbeit und dem Kurzarbeitergeld (20,7\%). Unterschiede ergeben sich hinsichtlich der Relevanz von KfW-Sonderprogramm und Überbrückungshilfe. Während im Baugewerbe das KfWSonderprogramm (3,8 \%) eine wichtigere Rolle als die Überbrückungshilfe $(1,8 \%)$ spielt, ist es in den anderen Industrien genau umgekehrt. 
Tabelle 4: Soforthilfe Corona und Kurzarbeit auch im Baugewerbe die wichtigsten Maßnahmen

Baugewerbe

Andere Industrien

\begin{tabular}{lccc|ccc} 
Anteil der Unternehmen (\%) & Durchschnitt & S.F. & $N$ & Durchschnitt & S.F. & $N$ \\
\hline Soforthilfe Corona & 29,5 & 3,17 & 459 & 47,7 & 1,13 & 8.489 \\
& $(27,4)$ & $(3,33)$ & $(694)$ & $(45,4)$ & $(1,02)$ & $(11.137)$ \\
& 1,8 & 0,93 & 459 & 11,2 & 0,7 & 8.489 \\
Überbrückungshilfe & $(1)$ & $(0,79)$ & $(694)$ & $(5,7)$ & $(0,46)$ & $(11.137)$ \\
Kurzarbeit und & 20,7 & 2,76 & 459 & 29,4 & 1,01 & 8.489 \\
Kurzarbeitergeld & $(18,2)$ & $(2,82)$ & $(694)$ & $(25,9)$ & $(0,86)$ & $(11.137)$ \\
& 3,8 & 1,16 & 459 & 7,7 & 0,58 & 8.489 \\
KfW-Sonderprogramm & $(5,9)$ & $(1,87)$ & $(694)$ & $(6,9)$ & $(0,5)$ & $(11.137)$ \\
& & & & & &
\end{tabular}

S.F. bezeichnet den Standardfehler des Mittelwerts, N die Zahl der Beobachtungen. Die Werte in Klammern beziehen sich auf die jeweiligen Ergebnisse der ersten Befragungswelle.

Ein ähnliches Bild ergibt sich, wenn man die beantragten oder erhaltenen steuerlichen Maßnahmen in den Blick nimmt. Auch hier haben durchschnittlich wesentlich weniger Unternehmen im Baugewerbe steuerliche Maßnahmen beantragt oder erhalten (19\%), als dies in den anderen Industrien der Fall ist (30 \%). Im Vergleich zu den nicht-steuerlichen Maßnahmen fällt auf, dass die steuerlichen Maßnahmen eine weniger bedeutende Rolle spielen, sowohl im Baugewerbe als auch bei den anderen Industrien.

\section{Auch bei den steuerlichen Maßnahmen profitieren durchschnittlich weniger Unternehmen des Baugewerbes}

Steuerliche Maßnahmen beantragt oder erhalten:

\section{$30 \%$}

der anderen Industrien

\section{$19 \%$}

des Baugewerbes

Die nachfolgende Tabelle 5 zeigt die vier steuerlichen Maßnahmen, die am häufigsten von Unternehmen genannt bzw. in Anspruch genommen wurden, sowie den jeweiligen Anteil der Unternehmen. Sowohl im Baugewerbe als auch in den anderen Industrien ist die Stundung von Steuerzahlungen die am häufigsten in Anspruch genommene steuerliche Maßnahme, gefolgt von der Erstattung bereits geleisteter Steuervorauszahlungen. Auffällig ist, dass es bei den anderen steuerlichen Maßnahmen (Verlustrücktrag und das Aussetzen von Vollstreckungsmaßnahmen) keine wesentlichen Unterschiede mehr zwischen dem Baugewerbe und den anderen Industrien gibt. 
Tabelle 5: Steuerstundung und Erstattung geleisteter Steuervorauszahlungen auch im Baugewerbe die wichtigsten Maßnahmen

Baugewerbe

Andere Industrien

\begin{tabular}{lccc|ccc} 
Anteil der Unternehmen (\%) & Durchschnitt & S.F. & N & Durchschnitt & S.F. & $N$ \\
\hline Erstattung geleisteter & 6 & 1,67 & 458 & 10,7 & 0,7 & 8.334 \\
Steuervorauszahlungen & $(8,6)$ & $(2,07)$ & $(683)$ & $(13,5)$ & $(0,69)$ & $(10.901)$ \\
& 2,2 & 0,95 & 458 & 3 & 0,34 & 8.334 \\
Sofortiger Verlustrücktrag & $(1,9)$ & $(0,84)$ & $(683)$ & $(5,7)$ & $(0,45)$ & $(10.901)$ \\
& 12,1 & 2,23 & 458 & 20,9 & 0,95 & 8.334 \\
Stundung von Steuerzahlung & $(17,8)$ & $(2,93)$ & $(683)$ & $(23,2)$ & $(0,9)$ & $(10.901)$ \\
Aussetzen & 1,3 & 0,69 & 458 & 2,1 & 0,3 & 8.334 \\
Vollstreckungsmaßnahmen & $(5,8)$ & $(1,91)$ & $(683)$ & $(3,5)$ & $(0,38)$ & $(10.901)$ \\
\hline
\end{tabular}

S.F. bezeichnet den Standardfehler des Mittelwerts, N die Zahl der Beobachtungen. Die Werte in Klammern beziehen sich auf die jeweiligen Ergebnisse der ersten Befragungswelle.

Im Baugewerbe erwarten durchschnittlich weniger Unternehmen, dass die staatlichen Maßnahmen ausreichen

Wir erwarten, dass die staatlichen Maßnahmen zum Überleben der Corona-Krise ausreichen:

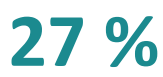

des Baugewerbes

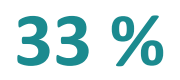

der anderen Industrien

Im Baugewerbe erwarten $27 \%$ der Unternehmen, dass die staatlichen Maßnahmen ausreichen. Der Anteil liegt damit deutlich unter dem Anteil, den die Befragung bei den Unternehmen in den anderen Industrien ergeben hat (33\%). Das ist insbesondere vor folgendem Hintergrund interessant: 1) Die Unternehmen des Baugewerbes sind besser durch die Corona-Krise gekommen. 2) Es haben durchschnittlich weniger Unternehmen aus dem Baugewerbe staatliche Maßnahmen beantragt oder erhalten. Der zweite Punkt könnte darauf hindeuten, dass die staatlichen Maßnahmen für das Baugewerbe nicht zielgerichtet waren und bspw. bestimmte Kriterien von den Unternehmen nicht erfüllt wurden. Auch die geringere Zufriedenheit im Baugewerbe mit den staatlichen Maßnahmen (s. oben) könnte darauf hinweisen. Vor diesem Hintergrund könnte ein Blick auf mögliche Hürden bei der Beantragung und dem Erhalt staatlicher Mittel Erklärungen liefern. Die nachfolgende Tabelle 6 zeigt die vier größten Hürden gemessen an der Häufigkeit der Nennung durch Unternehmen. 
Tabelle 6: Durchschnittlich weniger Unternehmen aus dem Baugewerbe berichten von konkreten Hürden bei der Beantragung

\begin{tabular}{lccc|ccc} 
& \multicolumn{3}{c}{ Baugewerbe } & \multicolumn{3}{c}{ Andere Industrien } \\
Anteil der Unternehmen (\%) & Durchschnitt & S.F. & N & Durchschnitt & S.F. & $N$ \\
\hline $\begin{array}{l}\text { Zu viel administrativer } \\
\text { Aufwand }\end{array}$ & 32,0 & 3,17 & 459 & 44,1 & 1,14 & 8.350 \\
Zu späte Auszahlung der Hilfen & 15,3 & 2,39 & 459 & 24,0 & 0,98 & 8.350 \\
Kriterien nicht erfüllt & 24,3 & 2,92 & 459 & 34,2 & 1,09 & 8.350 \\
Nicht bekannt & 47,6 & 3,40 & 459 & 29,4 & 1,02 & 8.350 \\
\hline
\end{tabular}

S.F. bezeichnet den Standardfehler des Mittelwerts, N die Zahl der Beobachtungen.

Insgesamt berichtet ein wesentlich geringerer Anteil der Unternehmen aus dem Baugewerbe von administrativen Hürden oder einer zu späten Auszahlung der Hilfen. Auch bei den zu erfüllenden Kriterien berichten durchschnittlich weniger Unternehmen aus dem Baugewerbe von Hürden als bei den anderen Industrien, was obige Vermutung nicht bestätigt.

\section{Maßnahmen zur Deckung der Mehrbelastung}

Neben der Inanspruchnahme von staatlichen Maßnahmen können auch interne Maßnahmen ergriffen werden, um die Mehrbelastung durch die Corona-Krise zu decken. Tabelle 7 zeigt die ergriffenen Maßnahmen, die von Unternehmen am häufigsten genannt wurden. Verglichen mit den anderen Industrien haben im Baugewerbe durchschnittlich weniger Unternehmen angegeben, dass sie weitere Maßnahmen zur Deckung der Mehrbelastung ergriffen haben. Vor dem Hintergrund der oben beschriebenen Beobachtung, dass Unternehmen des Baugewerbes besser durch die Corona-Krise gekommen sind, könnte dies darauf hindeuten, dass weniger Maßnahmen zur Deckung von potenziellen Mehrbelastungen ergriffen werden mussten, da das Baugewerbe insgesamt weniger stark von der Krise getroffen wurde. Dennoch haben 27,5\% der Unternehmen im Baugewerbe angegeben, dass sie Löhne und Boni reduziert haben, um der Krise entgegenzuwirken. Das ist auch bei den anderen Industrien die wichtigste Maßnahme $(28,4 \%)$, gefolgt von den Preiserhöhungen (22,9\%). Interessanterweise gibt es bei den sonstigen Maßnahmen zur Deckung der Mehrbelastung bis auf die Reduktion einbehaltener Gewinne keine wesentlichen Unterschiede zwischen Unternehmen des Baugewerbes und den Unternehmen der anderen Industrien. 
Tabelle 7: Weniger Unternehmen des Baugewerbes ergreifen sonstige Maßnahmen

\begin{tabular}{lccc|ccc} 
Anteil der Unternehmen (\%) & Durchschnitt & S.F. & $N$ & Durchschnitt & S.F. & $N$ \\
\hline Reduktion Löhne/Boni & 27,5 & 3,04 & 462 & 28,4 & 0,99 & 8.343 \\
& $(31,5)$ & $(3,44)$ & $(694)$ & $(31,6)$ & $(0,98)$ & $(9.763)$ \\
Abbau Arbeitsplätze & 15,7 & 2,56 & 462 & 17,4 & 0,84 & 8.343 \\
& $(8,9)$ & $(2,15)$ & $(694)$ & $(13,1)$ & $(0,69)$ & $(9.763)$ \\
Reduktion Ausschüttung & 13,9 & 2,25 & 462 & 16,1 & 2,25 & 8.343 \\
Anteilseigner & $(24,4)$ & $(3,27)$ & $(694)$ & $(16,6)$ & $(0,67)$ & $(9.763)$ \\
Reduktion einbehaltener & 11,8 & 2,04 & 462 & 20,2 & 0,95 & 8.343 \\
Gewinne & $(15,4)$ & $(2,73)$ & $(694)$ & $(19,4)$ & $(0,86)$ & $(9.763)$ \\
& 22,9 & 2,84 & 462 & 22,9 & 0,99 & 8.343 \\
Preiserhöhungen & $(30,3)$ & $(3,47)$ & $(694)$ & $(21,3)$ & $(0,9)$ & $(9.763)$
\end{tabular}

S.F. bezeichnet den Standardfehler des Mittelwerts, N die Zahl der Beobachtungen. Die Werte in Klammern beziehen sich auf die jeweiligen Ergebnisse der ersten Befragungswelle. 


\section{II - Ergebnisse einzelner Wirtschaftsyweige im Baugewerbe}

Siehe Abschnitt I. zur Datengrundlage.

Dem Gesetz über die Statistik im Produzierenden Gewerbe (ProdGewG) sowie Publikationen des Statistischen Bundesamtes folgend, ${ }^{1}$ wird für den Zweck der vorliegenden Analyse zwischen Ausbaugewerbe und Bauhauptgewerbe unterschieden. Dem Bauhauptgewerbe sind die WZ2008-Gruppen 41.2 "Bau von Gebäuden", 42.1 "Bau von Straßen und Bahnverkehrsstrecken", 42.2 "Leitungstiefbau und Kläranlagenbau", 42.9 "Sonstiger Tiefbau", 43.1 "Abbrucharbeiten und vorbereitende Baustellenarbeiten" und 43.9 "Sonstige spezialisierte Bautätigkeiten" zugerechnet. Unter Ausbaugewerbe werden die WZ-2008-Gruppen 43.2 "Bauinstallation" und 43.3 "Sonstiger Ausbau" geführt. Die zu den Bauträgern zählende WZ-2008-Gruppe 41.1 "Erschließung von Grundstücken; Bauträger" ist aus den Betrachtungen ausgeschlossen.

\section{Kennzahlen: Bauhauptgewerbe vs. Ausbaugewerbe}

Die folgende Tabelle zeigt eine Gegenüberstellung wesentlicher Kennzahlen des Ausbau- und Bauhauptgewerbes. Wie bereits in 1.1 dargelegt, geben die Veränderungen in Prozent jeweils die Steigerung / den Rückgang der Kennzahlen im Vergleich zum Jahresbeginn 2020 wieder. Während Betriebe des Ausbaugewerbes Umsatzeinbrüche von 3,1 \% im Vergleich zum Vorjahr vermelden, sind die Umsätze der Betriebe des Bauhauptgewerbes im Mittel leicht gestiegen. Anders verhält es sich bei den Gewinnen: Hier verzeichnet der Teil der im Bauhauptgewerbe tätigen Unternehmen stärkere Einbußen als Unternehmen des Ausbaugewerbes. Obwohl $19 \%$ der Unternehmen des Ausbaugewerbes und $8 \%$ der Unternehmen des Bauhauptgewerbes angeben, Mitarbeiter entlassen $z u$ haben (siehe unten), sind die Mitarbeiterzahlen im Baugewerbe insgesamt gestiegen. Im Ausbaugewerbe ist die Zahl der Beschäftigten im Vorjahresvergleich um 0,9\% gestiegen, im Bauhauptgewerbe um 5,6\%. Verglichen mit den Unterschieden zwischen anderen Industrien und dem Baugewerbe fallen die Unterschiede innerhalb des Baugewerbes klein aus.

1 Vgl. etwa „Lange Reihen der jährlichen Betriebserhebungen im Bauhaupt- und Ausbaugewerbe / Bauträger" - 2020, Statistisches Bundesamt (Destatis) 2021. 
Tabelle 8: Wachsende Mitarbeiterzahlen im Ausbau- und Bauhauptgewerbe bei leicht rückläufiger Gewinnentwicklung

\begin{tabular}{lccc|ccc} 
& \multicolumn{3}{c}{ Ausbaugewerbe } & \multicolumn{3}{c}{ Bauhauptgewerbe } \\
& Durchschnitt & S.F. & $N$ & Durchschnitt & S.F. & $N$ \\
& & & & & & \\
& $-3,1$ & 3,35 & 167 & 1,5 & 2,45 & 267 \\
Umsatzveränderung ggü. & $(-1,8)$ & $(3,9)$ & $(159)$ & $(-1,3)$ & $(1,89)$ & $(494)$ \\
Vorjahr (in \%) & $-0,5$ & 3,39 & 166 & $-2,5$ & 2,5 & 261 \\
Gewinnveränderung ggü. & $(-0,9)$ & $(3,94)$ & $(147)$ & $(-0,7)$ & $(1,82)$ & $(476)$ \\
Vorjahr (in \%) & $-1,1$ & 3,27 & 167 & 0,8 & 2,36 & 264 \\
Liquiditätsveränderung ggü. & $(2,1)$ & $(3,68)$ & $(156)$ & $(1,6)$ & $(1,85)$ & $(487)$ \\
Vorjahr (in \%) & & & & & & \\
Veränderung der & 0,9 & 2,65 & 169 & 5,6 & 1,94 & 267 \\
$\begin{array}{l}\text { Mitarbeiteranzahl ggü. Vorjahr } \\
\text { (in \%) }\end{array}$ & $(7,9)$ & $(3,42)$ & $(158)$ & $(2,8)$ & $(1,48)$ & $(489)$ \\
& & & & & & \\
\hline
\end{tabular}

S.F. bezeichnet den Standardfehler des Mittelwerts, N die Zahl der Beobachtungen. Die Werte in Klammern beziehen sich auf die jeweiligen Ergebnisse der ersten Befragungswelle.

Die folgende Tabelle zeigt die von teilnehmenden Unternehmen berichtete, allgemeine Unsicherheit, mit der sich die Unternehmen im Mittel konfrontiert sehen sowie die erwartete Überlebensrate in der Branche und die erwartete Veränderung des Bruttoinlandsprodukts (BIP) im Jahr 2021. Insgesamt ist bezüglich der allgemeinen Auffassung und Einschätzung ein homogenes Stimmungsbild innerhalb des Baugewerbes zu beobachten. Etwaige Unterschiede spielen sich im Bereich von Nachkommastellen ab. Mit Blick auf die durch die gegenwärtige Pandemie beeinflusste Wirtschaftslage sind die Erwartungen und Einschätzungen der Unternehmen des Ausbau- sowie Bauhauptgewerbes im Mittel ähnlich verhalten. Auf einer Skala von 1 (sehr gering) - 5 (sehr hoch) bewerten Unternehmen des Ausbaugewerbes die Unsicherheit im Mittel mit 3,6 (Baugewerbe: 3,5). Unternehmen in beiden Bereichen teilen eine im Mittel negativere Erwartung bezüglich der Entwicklung des Bruttoinlandsprodukts verglichen mit Unternehmen sonstiger Industrien (siehe oben). Geschätzte Überlebensraten der Unternehmen innerhalb der jeweiligen Branche sind sich ebenfalls ähnlich und liegen bei ungefähr $80 \%$, die Zufriedenheit mit staatlichen Maßnahmen ist deckungsgleich. 
Tabelle 9: Deckungsgleiche Auffassungen zu allgemeiner Unsicherheit, erwarteten Überlebensraten sowie zur gesamtwirtschaftlichen Entwicklung

\begin{tabular}{|c|c|c|c|c|c|c|}
\hline & \multicolumn{3}{|c|}{ Ausbaugewerbe } & \multicolumn{3}{|c|}{ Bauhauptgewerbe } \\
\hline & Durchschnitt & S.F. & $N$ & Durchschnitt & S.F. & $\mathrm{N}$ \\
\hline $\begin{array}{l}\text { Allgemeine Unsicherheit } \\
\text { (Skala: } 1-5 \text { ) }\end{array}$ & $\begin{array}{c}3,6 \\
(n / a)\end{array}$ & $\begin{array}{c}0,1 \\
(\mathrm{n} / \mathrm{a})\end{array}$ & $\begin{array}{l}169 \\
(\mathrm{n} / \mathrm{a})\end{array}$ & $\begin{array}{l}3,5 \\
\text { (n/a) }\end{array}$ & $\begin{array}{l}0,07 \\
(\mathrm{n} / \mathrm{a})\end{array}$ & $\begin{array}{l}265 \\
(\mathrm{n} / \mathrm{a})\end{array}$ \\
\hline $\begin{array}{l}\text { Erwartete Überlebensrate in } \\
\text { eigener Branche (in \%) }\end{array}$ & $\begin{array}{l}80,8 \\
(84,4)\end{array}$ & $\begin{array}{c}1,77 \\
(1,94)\end{array}$ & $\begin{array}{c}175 \\
(153)\end{array}$ & $\begin{array}{c}80,3 \\
(84,6)\end{array}$ & $\begin{array}{c}1,42 \\
(0,85)\end{array}$ & $\begin{array}{c}284 \\
(484)\end{array}$ \\
\hline $\begin{array}{l}\text { Erwartete BIP-Veränderung in } \\
2021 \text { (in \%) }\end{array}$ & $\begin{array}{l}-8,9 \\
(-0,5)\end{array}$ & $\begin{array}{l}1,11 \\
(0,51)\end{array}$ & $\begin{array}{c}115 \\
(132)\end{array}$ & $\begin{array}{l}-8,8 \\
(-1,6)\end{array}$ & $\begin{array}{c}0,82 \\
(0,26)\end{array}$ & $\begin{array}{l}220 \\
(442)\end{array}$ \\
\hline $\begin{array}{l}\text { Zufriedenheit mit staatl. } \\
\text { Maßnahmen ggü. Vorjahr } \\
\text { (Skala: 0-4) }\end{array}$ & $\begin{array}{l}1,6 \\
(n / a)\end{array}$ & $\begin{array}{l}0,13 \\
(n / a)\end{array}$ & $\begin{array}{l}93 \\
(\mathrm{n} / \mathrm{a})\end{array}$ & $\begin{array}{l}1,6 \\
(n / a)\end{array}$ & $\begin{array}{l}0,12 \\
(\mathrm{n} / \mathrm{a})\end{array}$ & $\begin{array}{l}105 \\
(\mathrm{n} / \mathrm{a})\end{array}$ \\
\hline
\end{tabular}

S.F. bezeichnet den Standardfehler des Mittelwerts, N die Zahl der Beobachtungen. Die Werte in Klammern beziehen sich auf die jeweiligen Ergebnisse der ersten Befragungswelle.

Innerhalb beider Branchen verzeichnen große Unternehmen höhere Umsatzeinbrüche, allerdings sind auch kleine Unternehmen des Ausbaugewerbes von einem Umsatzrückgang betroffen. Das größte Umsatzwachstum innerhalb der dargestellten Größenkategorien verzeichnen mittelgroße Unternehmen mit mehr als 9, weniger als 20 Mitarbeitern des Bauhauptgewerbes, aber auch mittelgroße Unternehmen des Ausbaugewerbes geben eine positive Entwicklung an.

Tabelle 10: Große Betriebe sowie kleine Betriebe des Ausbaugewerbes sind stärker von einem rückläufigen Umsatz betroffen

$$
\text { Ausbaugewerbe Bauhauptgewerbe }
$$

\begin{tabular}{lccc|ccc} 
Umsatzveränderung ggü. Vorjahr (\%) & Durchschnitt & S.F. & N & Durchschnitt & S.F. & N \\
\hline O bis 9 Mitarbeiter & $-3,4$ & 3,68 & 102 & 1,3 & 3,02 & 144 \\
& $(-2,4)$ & $(4,3)$ & $(83)$ & $(-3,3)$ & $(2,29)$ & $(288)$ \\
& 1,8 & 6,49 & 34 & 5,4 & 3,87 & 59 \\
10 bis 19 Mitarbeiter & $(7,6)$ & $(5,85)$ & $(36)$ & $(7,9)$ & $(3,7)$ & $(84)$ \\
& -4 & 6,23 & 31 & $-0,8$ & 2,77 & 64 \\
Mehr als 20 Mitarbeiter & $(-0,9)$ & $(3,87)$ & $(40)$ & $(6,1)$ & $(2,86)$ & $(121)$ \\
\hline
\end{tabular}

S.F. bezeichnet den Standardfehler des Mittelwerts, N die Zahl der Beobachtungen. Die Werte in Klammern beziehen sich auf die jeweiligen Ergebnisse der ersten Befragungswelle. 


\section{Inanspruchnahme von staatlichen Maßnahmen}

Unterschiede in der Beantragung staatlicher Maßnahmen zwischen Ausbau- und Bauhauptgewerbe sind vor allem im Bereich der nicht-steuerlichen Maßnahmen zu verorten. Im Bereich der steuerlichen Maßnahmen zeigt sich eine größere Ähnlichkeit der von den jeweiligen Branchen angefragten Maßnahmen.

Betriebe des Ausbaugewerbes nutzen nicht-steuerliche Maßnahmen stärker als Betriebe des Bauhauptgewerbes

Nicht-steuerliche Maßnahmen beantragt oder erhalten:

\section{$47 \% \quad 36 \%$}

des Ausbaugewerbes

des Bauhauptgewerbes

Tabelle 11: Lediglich die Überbrückungshilfe ist für das Bauhauptgewerbe relevanter

Ausbaugewerbe $\quad$ Bauhauptgewerbe

\begin{tabular}{lccc|ccc} 
Anteil der Unternehmen (\%) & Durchschnitt & S.F. & $N$ & Durchschnitt & S.F. & $N$ \\
\hline Soforthilfe Corona & 31,9 & 4,16 & 175 & 22,7 & 2,87 & 284 \\
& $(27,6)$ & $(4,42)$ & $(168)$ & $(26,8)$ & $(2,16)$ & $(526)$ \\
& 1,7 & 1,2 & 175 & 2,2 & 1,03 & 284 \\
Überbrückungshilfe & $(1,1)$ & $(1,05)$ & $(168)$ & $(0,8)$ & $(0,45)$ & $(526)$ \\
& 21,9 & 3,63 & 175 & 17,2 & 2,54 & 284 \\
& $(17,9)$ & $(3,74)$ & $(168)$ & $(19,3)$ & $(1,86)$ & $(526)$ \\
Kurzarbeit und Kurzarbeitergeld & 3,9 & 1,52 & 175 & 3,5 & 1,04 & 284 \\
& $(6,9)$ & $(2,51)$ & $(168)$ & $(2,9)$ & $(0,78)$ & $(526)$
\end{tabular}

S.F. bezeichnet den Standardfehler des Mittelwerts, N die Zahl der Beobachtungen. Die Werte in Klammern beziehen sich auf die jeweiligen Ergebnisse der ersten Befragungswelle.

Nicht-steuerliche Maßnahmen wurden von $47 \%$ der Betriebe des Ausbaugewerbes in Anspruch genommen, von nur $36 \%$ der Betriebe des Bauhauptgewerbes. Ein kompatibles Bild zeigt sich bei der Betrachtung der einzelnen Maßnahmen. Mit Ausnahme der Überbrückungshilfe wurden die einzelnen nicht-steuerlichen Maßnahmen stärker von Betrieben des Ausbaugewerbes nachgefragt, bei der Soforthilfe Corona beträgt der Unterschied 9,2 \%, im Falle von Kurzarbeit und Kurzarbeitergeld 4,7\%. Die Unterschiede bezüglich der Inanspruchnahme von Überbrückungshilfe und KfW-Sonderprogramm sind hingegen sehr gering. 


\section{Jedes fünfte Unternehmen des Ausbau- wie Bauhauptgewerbes beansprucht steuerliche Maßnahmen}

Steuerliche Maßnahmen beantragt oder erhalten:

\section{$19 \% \quad 18 \%$}

des Ausbaugewerbes

des Hauptbaugewerbes

Insgesamt spielen steuerliche Maßnahmen für Unternehmen des Baugewerbes, wie im ersten Teil des Berichts dargelegt, eine nachrangige Rolle. 19 \% der Unternehmen des Ausbaugewerbes, respektive $18 \%$ der Unternehmen des Bauhauptgewerbes haben diese Maßnahmen in Anspruch genommen. Bis auf das Aussetzen von Vollstreckungsmaßnahmen wurden alle steuerlichen Maßnahmen von Betrieben des Ausbaugewerbes stärker beansprucht als von Betrieben des Bauhauptgewerbes. Die Unterschiede sind jedoch gering. Die Möglichkeit, Steuerzahlungen zu stunden, sticht aus den Maßnahmen heraus. Diese Möglichkeit haben mehr als $10 \%$ der Unternehmen beider Branchen wahrgenommen.

Tabelle 12: Keine wesentlichen Unterschiede bei den steuerlichen Maßnahmen

\begin{tabular}{lccc|ccc} 
& \multicolumn{3}{c}{ Ausbaugewerbe } & \multicolumn{3}{c}{ Bauhauptgewerbe } \\
Anteil der Unternehmen (\%) & Durchschnitt & S.F. & $N$ & Durchschnitt & S.F. & $N$ \\
\hline Erstattung geleisteter & & & & & & \\
Steuervorauszahlungen & $(8,6)$ & $(2,76)$ & $(164)$ & $(8,6)$ & $(1,33)$ & $(519)$ \\
& 2,3 & 1,24 & 175 & 2 & 0,93 & 283 \\
Sofortiger Verlustrücktrag & $(1,5)$ & $(1,1)$ & $(164)$ & $(3,2)$ & $(0,85)$ & $(519)$ \\
& 12,6 & 2,93 & 175 & 10,8 & 2,05 & 283 \\
Stundung von Steuerzahlung & $(18,3)$ & $(3,91)$ & $(164)$ & $(16,4)$ & $(1,78)$ & $(519)$ \\
Aussetzen & 0,9 & 0,85 & 175 & 2,4 & 0,85 & 283 \\
Vollstreckungsmaßnahmen & $(6,7)$ & $(2,56)$ & $(164)$ & $(3,1)$ & $(0,85)$ & $(519)$ \\
\hline
\end{tabular}

S.F. bezeichnet den Standardfehler des Mittelwerts, N die Zahl der Beobachtungen. Die Werte in Klammern beziehen sich auf die jeweiligen Ergebnisse der ersten Befragungswelle.

Vergleichbare Einschätzungen über die Hinlänglichkeit der staatlichen Maßnahmen im Ausbau- und Bauhauptgewerbe

Erwarten, dass die staatlichen Maßnahmen zum Überleben der Corona-Krise nicht ausreichen:

\section{$73 \% \quad 69 \%$}


$73 \%$ der Unternehmen des Ausbaugewerbes halten die Maßnahmen für nicht ausreichend. Ähnliches gilt für das Bauhauptgewerbe, hier sind es $69 \%$ der Unternehmen. Diese Situation könnte mit etwaigen Hürden bei Beantragung und Erhalt staatlicher Mittel in Verbindung stehen. So werden administrativer Aufwand und späte Auszahlung der Hilfen von etwa $34 \%$ der Betriebe des Bauhauptgewerbes und von etwa $31 \%$ der Betriebe des Ausbaugewerbes als Haupthürden angeführt. Ungefähr ein Viertel der Unternehmen beider Branchen erfüllt die Voraussetzungen zur Beantragung der Maßnahmen nicht.

Tabelle 13: Hürden bei der Beantragung und dem Erhalt staatlicher Mittel nach Bauhauptgewerbe und Ausbaugewerbe

\begin{tabular}{lccc|ccc} 
& \multicolumn{3}{c}{ Ausbaugewerbe } & \multicolumn{3}{c}{ Bauhauptgewerbe } \\
Anteil der Unternehmen (\%) & Durchschnitt & S.F. & N & Durchschnitt & S.F. & N \\
\hline Zu viel administrativer Aufwand & 31,3 & 4,13 & 175 & 34,1 & 3,14 & 284 \\
Zu späte Auszahlung der Hilfen & 14,1 & 3,08 & 175 & 18,8 & 2,64 & 284 \\
Kriterien nicht erfüllt & 24,2 & 3,81 & 175 & 24,8 & 2,90 & 284 \\
Nicht bekannt & 49,5 & 4,44 & 175 & 42,1 & 3,29 & 284 \\
\hline
\end{tabular}

\section{Maßnahmen zur Deckung der Mehrbelastung}

Verglichen mit anderen Industrien verzeichnen Unternehmen der Baubranche im Mittel eine geringere Belastung in Form von Umsatz- oder Gewinneinbrüchen. Dennoch haben auch Unternehmen des Baugewerbes Maßnahmen zur Deckung der Mehrbelastung getroffen. Größere Abweichungen zwischen Ausbau- und Bauhauptgewerbe gibt es diesbezüglich insbesondere im Bereich des Abbaus von Arbeitsplätzen. Während fast jedes fünfte Unternehmen des Ausbaugewerbes zu diesem Mittel griff, waren es im Bauhauptgewerbe weniger als $10 \%$ der Unternehmen. Sowohl im Ausbau- als auch im Bauhauptgewerbe tätige Betriebe konnten Preiserhöhungen durchsetzen. Dieses Mittel war für beide Branchen nach der Reduktion der Löhne/Boni die Maßnahme, die am häufigsten durchgesetzt wurde. 


\title{
Tabelle 14: Fast jedes fünfte Unternehmen des Ausbaugewerbes musste Arbeitsplätze abbauen
}

\author{
Ausbaugewerbe
}

Bauhauptgewerbe

\begin{tabular}{lccc|ccc} 
Anteil der Unternehmen (\%) & Durchschnitt & S.F. & $N$ & Durchschnitt & S.F. & $N$ \\
\hline Reduktion Löhne/Boni & 27,9 & 3,98 & 177 & 26,4 & 2,89 & 285 \\
& $(32)$ & $(4,58)$ & $(168)$ & $(30,2)$ & $(2,19)$ & $(526)$ \\
Abbau Arbeitsplätze & 18 & 3,39 & 177 & 9 & 1,86 & 285 \\
& $(9,1)$ & $(2,86)$ & $(168)$ & $(8,3)$ & $(1,33)$ & $(526)$ \\
Reduktion Ausschüttung & 12,9 & 2,91 & 177 & 16,7 & 2,43 & 285 \\
Anteilseigner & $(25,3)$ & $(4,35)$ & $(168)$ & $(21,8)$ & $(1,99)$ & $(526)$ \\
& 10,4 & 2,6 & 177 & 15,8 & 2,46 & 285 \\
& $(15,9)$ & $(3,63)$ & $(168)$ & $(14,1)$ & $(1,67)$ & $(526)$ \\
Reduktion einbehaltener Gewinne & 22,9 & 3,7 & 177 & 23,2 & 2,84 & 285 \\
& $(32,6)$ & $(4,62)$ & $(168)$ & $(23,9)$ & $(2,02)$ & $(526)$
\end{tabular}

S.F. bezeichnet den Standardfehler des Mittelwerts, N die Zahl der Beobachtungen. Die Werte in Klammern beziehen sich auf die jeweiligen Ergebnisse der ersten Befragungswelle.

„Da wir in der Baubranche tätig sind, gehe ich davon aus, dass wir erst später von der Krise betroffen sein werden."

Unternehmen der Baubranche

„[...] Unser Unternehmen ist auch wegen seiner Corona-Teststrategie gut durch die Krise gekommen. Dass diese Tests lange nur an Ärzte ausgegeben werden durften und z.B. vom BMG aktiv von Selbsttests abgeraten wurde, Massentests in Hotspots und kritischen Branchen unterbleiben, ist unverantwortlich. [...]."

Unternehmen der Baubranche

„Unser Unternehmen ist in soweit belastet, dass wir zur Baubranche gehören und sehr viel zusätzliche Aufträge hatten. Jeder hat einen Garten, Terrasse, Schwimmbäder, Kellerreinigung u.v.m. Kein Urlaub deshalb war der Garten und Wohnbereich hauptsächlich Freizeitbeschäftigung. Wir haben von der Krise profitiert. Ich bin der Meinung, dass die Verschiebung der Insolvenzen nicht richtig ist, da viele Firmen bereits insolvent sind und dies nur eine Verzögerung bedeutet. [...]."

Unternehmen der Baubranche

„Wir sind in der Baubranche tätig und sind bisher von der Corona-Krise kaum betroffen, die Probleme, die dieses Jahr auftraten mit Gewinnreduzierung sind nicht auf die aktuelle Corona-Pandemie zurückzuführen."

Unternehmen der Baubranche 
Das German Business Panel ist ein langfristiges Befragungspanel des DFG-geförderten überregionalen Projektes „Accounting for Transparency"

SFB/Transregio 266

ACCOUNTING FOR TRANSPARENCY

www.accounting-for-transparency.de

Project C01: www.gbpanel.org

D $\begin{aligned} & \text { Deutsche } \\ & \text { Forschungsgemeinschaft }\end{aligned}$

Gefördert durch die Deutsche Forschungsgemeinschaft (DFG) - Collaborative Research Center

(SFB/TRR) Project-ID 403041268 - TRR 266 Accounting for Transparency 